\title{
PRIMEIRO REGISTRO DE Schizophragma bicolor (DOZIER, 1932) (HYMENOPTERA: MYMARIDAE) NO BRASIL
}

\author{
Rodrigo Souza Santos 1 , Valmir Antonio Costa ${ }^{2}$
}

${ }^{1}$ FCAV/UNESP, Faculdade de Ciências Agrárias e Veterinárias, Via de Acesso Prof. Paulo Donato Castellane s/n, CEP: 14884-900, Jaboticabal, SP, Brasil, E-mail: santos_rss@hotmail.com

${ }^{2}$ Instituto Biológico, Agência Paulista de Tecnologia dos Agronegócios, Rodovia Heitor Penteado, km 3, Caixa Postal 70, CEP: 13001-970, Campinas, SP.

\section{RESUMO}

O objetivo desta comunicação científica é registrar a ocorrência de Schizophragma bicolor (Dozier) (Hymenoptera: Mymaridae) no Brasil. O trabalho objetivava verificar parasitóides de ovos endofíticos de Leptopharsa heveae Drake \& Poor (Hemiptera: Tingidae), em folíolos maduros de seringueira na fazenda da empresa "Plantações E. Michelin", Itiquira, MT. Semanalmente, folíolos eram coletados, lavados em solução de hipoclorito de sódio a 1,5\%, secados, acondicionados em sacos plásticos, inflados com auxílio de um compressor de ar, selados e colocados em sala climatizada a $25 \pm 1{ }^{\circ} \mathrm{C}$. Após cinco dias o material era examinado sob microscópio estereoscópico, sendo contabilizado o número de parasitóides emergidos. Além dos parasitóides de ovos de $L$. heveae, foi obtido um total de 29 exemplares de $S$. bicolor dos folíolos de seringueira. Com a metodologia utilizada nos ensaios, as posturas externas à superfície dos folíolos são eliminadas, mas não as posturas endofíticas de outros insetos, além daquelas de $L$. heveae. Como foi observada a presença de três ninfas de cicadelídeos não identificados nos ensaios, não é possível saber se $S$. bicolor estava associado a estas ou a $L$. heveae. Apesar disso, está registrada a primeira ocorrência deste mimarídeo no país.

Palavras-chave: Chalcidoidea, Cicadellidae, Hevea brasiliensis, Tingidae, parasitóide.

\section{FIRST RECORD OF Schizophragma bicolor (DOZIER, 1932) (HYMENOPTERA: MYMARIDAE) NO BRASIL}

\begin{abstract}
This paper deals with the record of Schizophragma bicolor (Dozier) (Hymenoptera: Mymaridae) in Brazil. The objective of the study was to survey parasitoids of Leptopharsa heveae Drake \& Poor (Hemiptera: Tingidae) eggs in mature leaves of rubber tree at the "Plantações E. Michelin" farm, at Itiquira, state of Mato Grosso, Brazil. The folioles were collected weekly, washed in sodium hypochlorite $1.5 \%$ solution, let to dry and disposed in plastic bags, which were inflated by an air compressor and then sealed and transferred to acclimatized room $\left(25 \pm 1{ }^{\circ} \mathrm{C}\right)$. Five days after, the material was examined under stereomicroscope and the number of adult parasitoids was registered. Besides the parasitoids of $L$. heveae eggs, 29 specimens of $S$. bicolor were obtained from the rubber tree folioles. The methodology employed in the study permits the elimination of external eggs, but preserves the endophytic egg masses, from $L$. heveae and other insects. As nymphs of unidentified leafhoppers (Hemiptera: Cicadellidae) were observed inside the bags, it is not possible to know if $S$. bicolor
\end{abstract}


was associated to the cicadellids or to the tingid. In spite of the association problem, this is the first occurrence of this mymarid in the country.

Key words: Chalcidoidea, Cicadellidae, Hevea brasiliensis, Tingidae, parasitoid.

\section{INTRODUÇÃO}

A família Mymaridae inclui 104 gêneros e 1430 espécies (NOYES, 2001), das quais 579 ocorrem na Região Neotropical e 73 no Brasil (NOYES, 2003). Assim, o Brasil tem registrado apenas $12,6 \%$ das espécies da Região Neotropical e $5,1 \%$ do total, valores muito baixos para um país cuja biodiversidade é considerada elevada.

Estes himenópteros são vulgarmente conhecidos, em inglês, como "fairyflies" (Yoshimoto, 1990). Todas as espécies de hábito conhecido são endoparasitóides de ovos de insetos. A maioria dos registros de hospedeiros corresponde a Auchenorrhyncha (Hemiptera), seguidos por outros Hemiptera (Coccoidea, Tingidae e Miridae), Coleoptera e Psocoptera. Noyes (2003), com base em Huber (1986), sugere que há preferência por ovos ocultos e que os parasitóides não apresentam grande especificidade de hospedeiro. Diversas espécies, quase todas dos gêneros Anaphes ou Anagrus, estão sendo utilizadas como agentes de controle biológico (SANCHES, 2000).

Atualmente, o gênero Schizophragma Oglobin, 1949 contém oito espécies, sendo duas de ocorrência no Brasil, S. basalis Oglobin, 1949 e S. latipennis (Crawford, 1913), cujos hospedeiros primários são hemípteros da família Membracidae (Hemiptera) (OGLOBIN, 1949, De SANTIS, 1979 e 1980, HUBER, 1987).

A distribuição da espécie $S$. bicolor (Dozier, 1932) está relatada para os seguintes países: Costa Rica, Guatemala, Haiti, Havai, México, Porto Rico, Trinidad \& Tobago e Estados Unidos, estando associada a cicadelídeos (Hemiptera) (De Santis \& Fidalgo, 1994, Dozier, 1932,
Thompson, 1958, Alyokhin et al., 2001, Yang et al., 2002, Viggiani, 2005).

Neste trabalho é feito o primeiro registro de $S$. bicolor no Brasil, no município de Itiquira, MT.

\section{MATERIAL E MÉTODOS}

O estudo foi conduzido entre outubro de 2005 a fevereiro de 2006 e de agosto de 2006 a janeiro de 2007, na fazenda da empresa "Plantações E. Michelin Ltda." (P.E.M.), município de Itiquira, MT (17'22'23's, 54\%44'23"W).

Semanalmente, folíolos maduros de cinco clones de seringueira (RRIM 600, PR 255, PB 235, PB 217 e GT 1) apresentando sintomas de ataque de Leptopharsa heveae Drake \& Poor, 1935 (Hemiptera: Tingidae) foram coletados em campo e levadas ao laboratório, onde foram lavados em solução de hipoclorito de sódio a 1,5\%, com auxílio de um pincel, para remoção de impurezas e ovos externos às folhas. Após, os folíolos foram enxaguados em água destilada por dois minutos e mantidos em papel absorvente até a secagem, seguindo a metodologia adaptada do trabalho de Costa et al. (2003).

Os pecíolos de cada folíolo foram inseridos em tubos plásticos contendo água destilada, tendo sua abertura lacrada com Parafilm $\mathrm{M}^{\circledR}$ para evitar o escoamento da mesma. Esse material (tubos + folíolos) foi colocado em sacos plásticos $(12 \times 30 \mathrm{~cm})$ identificados, inflados com um compressor de ar e selados com auxílio de uma seladora elétrica. Os sacos foram transferidos para sala climatizada a $25 \pm 1{ }^{\circ} \mathrm{C}$ e fotofase de 12 horas e pendurados em varais, com auxílio de prendedores. 
A avaliação procedeu-se cinco dias após a coleta, observando-se os sacos plásticos e folíolos sob microscópio estereoscópico, verificando a presença dos parasitóides emergidos.

\section{RESULTADOS E DISCUSSÃO}

Foi contabilizado um total de 29 exemplares de $S$. bicolor nos cinco clones estudados, sendo 15 no PB 217, 5 no PB 235 , 4 no RRIM 600, 4 no PR 255 e 1 no GT 1. Assim, registra-se pela primeira vez a ocorrência de S. bicolor no Brasil.

Este mimarídeo é relatado parasitando ovos dos cicadelídeos Empoasca fabalis DeLong (De Santis, 1979; Thompson, 1958) nas Américas e Sophonia rufofascia (Kuoh \& Kuoh) no Havai (Alyokhin et al., 2001, Yang et al., 2002).

No Brasil não há registros de Sophonia spp.; contudo, ocorrem várias espécies do gênero Empoasca, inclusive $E$. fabalis (Fernández, 2003). A metodologia utilizada nos ensaios elimina quaisquer posturas externas à superfície dos folíolos, mas não as posturas endofíticas de outros insetos, além das de $L$. heveae. Como foi observada a presença de três ninfas de cicadelídeos não identificados nos ensaios, não é possível afirmar se $S$. bicolor estava associado a estas ou a $L$. heveae. Desta forma, a associação de $S$. bicolor com ovos de $L$. heveae ou dos cicadelídeos encontrados ainda precisa ser confirmada. Não obstante, amplia-se com este trabalho o número de espécies de Mymaridae conhecidas para o país.

\section{REFERÊNCIAS BIBLIOGRÁFICAS}

ALYOKHIN, A.V.; YANG, P.J.; MESSING, R.H. 2001. Distribution and parasitism of Sophonia rufofascia (Homoptera: Cicadellidae) eggs in Hawaii. Annals of the Entomological Society of America, 94(5): 664-669.

COSTA, V.A.; PEREIRA, C. de F.; BATISTA FILHO, A. 2003. Observações preliminares sobre o parasitismo de ovos de Leptopharsa heveae (Hemiptera: Tingidae) em seringueira em Pindorama, SP. Arquivos do Instituto Biológico, 70(2): 205-206.

De SANTIS, L. 1979. Catalogo de los Himenópteros Calcidoideos de la América al sur de los Estados Unidos. Publicación Especial Comisión de Investigaciones Científicas. Provincia de Buenos Aires, 372.

De SANTIS, L. 1980. Catalogo de los Himenópteros de la serie Parasitica incluyendo Bethyloidea. Editora da Universidade Federal do Paraná, Curitiba, $149 \mathrm{p}$.

De SANTIS, L.; FIDALGO, P. 1994. Catalogo de Himenópteros Calcidoideos. Serie de la Academia Nacional de Agronomía y Veterinaria, 13: 126.

DOZIER, H.L. 1932, Descriptions of new Mymaridae egg parasites from Haiti and Puerto Rico. Journal of Department of Agriculture of Porto Rico, 16; 81-91.

FERNÁNDEZ, J.M.L. 2003. Aportación al conocimiento de la dinámica poblacional de Empoasca fabalis DeLong (Homoptera: Cicadellidae) sobre Ipomea (sic) batatas (L.) Poir en la Isla de la Palma-Islas Canarias-España, en condiciones de campo. Phytoma España, 154: 28-31.

HUBER, J.T. 1987. Review of Schizophragma Oglobin and the nonAustralian species of Stethynium Enok (Hymenoptera: Mymaridae). Canadian Entomologist, 119(9): 823-855.

NOYES, J.S. 2003. Universal Chalcidoidea Database. World Wide Web electronic publication. Informações disponíveis em: $<$ http://www.nhm.ac.uk/entomology/chal 
cidoids/index.html> Acesso em: 01 abr. 2008.

NOYES, J.S. 2001. Chalcidoidea 2001: biological and taxonomical information. The Natural History Museum, Londres, 1 CD-ROM, 2001. Informações disponíveis em: $<$ http://www.nhm.ac.uk/entomology/chal cidoids/tanaostigmatidae.html $>$ Acesso em: 01 abr. 2008.

OGLOBIN, A.A. 1949. Un nuevo género de Mymaridae de la Región Neotrópica. Notas del Museo de la Plata, Buenos Aires XIV (Zoología, nº 129): 345.

SANCHES, M.A. 2000. Parasitism of eggs of Gonipterus scutellatus and Gonipterus gibberus by the mymarid Anaphes nitens in Colombo, Paraná, Brasil. Arquivos do Instituto Biológico, 67: 77-82.

THOMPSON, W.R. 1958. A catalogue of the parasites and predators of insect pests. Section 2. Host parasite catalogue, part 5. Commonwealth Agricultural Bureau, Commonwealth Institute of Biological Control, 558.

VIGGIANI, G. 2005. A further contribution to the knowledge of the male genitalia in the Mymaridae (Hymenoptera:

Chalcidoidea). Bolletino del Laboratorio di Entomologia Agraria 'Filippo Silvestri', 59: 59-74.

YANG, P.; FOOTE, D.; ALYOKHIN, A.V.; LENZ, L.; MESSING, R.H. 2002. Distribution and abundance of mymarid parasitoids (Hymenoptera: Mymaridae) of Sophonia rufofascia Kuoh and Kuoh (Homoptera: Cicadellidae) in Hawaii. Biological Control, 23(3): 237-244.

YOSHIMOTO, C.M. 1990. A review of the genera of New World Mymaridae (Hymenoptera: Chalcidoidea). Flora \& Fauna Handbook $n^{0}$ 7. Sandhill Crane Press, Inc. Gainesville, Florida, $166 \mathrm{p}$. 